\title{
Calidad en el desempeño docente en la universidad nacional del Cusco
}

\author{
Mgt. Silvia Hidalgo Delgado \\ silviahidalgo@yahoo.com
}

https://orcid.org/0000-0003-0828-846X

Filiación Universidad César Vallejo

\section{RESUMEN}

La nueva normativa impuesta por el estado peruano, tras la promulgación de la ley $\mathrm{N}^{\mathrm{o}}$ 30220 nueva Ley Universitaria, con la que se inicia el proceso continuo de mejora de la calidad educativa de los claustros universitarios públicos como privados, considerados como entidades esenciales del progreso nacional, de la búsqueda y del conocimiento, las instituciones de formación superior de nuestra patria, las que deben buscar la calidad de sus procesos de sus escuelas profesionales, la cual deberá estar fundada en la obtención de la calidad de la enseñanza, el buen desempeño docente, búsqueda y excelentes servicios; conversar de calidad no significa un tema nuevo; sin embargo, si hablamos de estándares de calidad o normas internacionales de calidad, sí lo es; en la vida diaria de las universidades se genera una nueva y novedosa cultura organizacional todo ello enmarcado en la búsqueda de un servicio de calidad general, buen desempeño docente, las universidades de nuestro país se han imbuido, con bastante éxito, en la conducción de estándares internacionales con la finalidad de afianzar la calidad de su proceso formativo.

La interrupción provocada por la pandemia de la covid 19 significo un proceso de adaptación e innovación en los sistemas de enseñanza, desempeño docente en la virtualidad, lo cual significo avances y a su vez acentuar las brechas educativas pre existentes en nuestro país, lo más significativo esta referido a los estudiantes más vulnerables y para quienes son más privilegiados en cuanto a tecnología se refiere, ademásde otros indicadores educativos referidos a la permanencia en la universidad y la progresión, resaltando además la contradicción entre la actividad económica que se tiene en el país.

Palabras clave: estándares; pandemia; brechas educativas 


\title{
Quality in teaching performance in the national university of Cusco
}

\begin{abstract}
The new regulations imposed by the Peruvian state, after the enactment of Law No. 30220 new University Law, which begins the continuous process of improving the educational quality of public and private university cloisters, considered as essential entities of national progress, search and knowledge, higher education institutions of our country, which should seek the quality of their processes of their professional schools, which should be based on obtaining the quality of teaching, good teaching performance, search and excellent services; Talking about quality is not a new topic; however, if we talk about quality standards or international quality norms, it is; in the daily life of the universities a new and novel organizational culture is generated, all framed in the search for a general quality service, good teaching performance, the universities of our country have been imbued, quite successfully, in the conduction of international standards with the purpose of strengthening the quality of their formative process.

The interruption caused by the pandemic of covid 19 meant a process of adaptation and innovation in teaching systems, teaching performance in virtuality, which meant progress and in turn accentuate the pre-existing educational gaps in our country, the most significant is referred to the most vulnerable students and for those who are more privileged in terms of technology is concerned, in addition to other educational indicators referred to the permanence in the university and progression, also highlighting the contradiction between the economic activity that takes place in the country.
\end{abstract}

Key words: standards; pandemic; educational gaps.

Artículo recibido: 30 noviembre. 2021 Aceptado para publicación: 29 diciembre 2021 Correspondencia: silviahidalgo@yahoo.com Conflictos de Interés: Ninguna que declarar 


\section{INTRODUCCIÓN}

Ministerio de Educación (2020), entidad estatal que refiere que, nuestro país, celebró el bicentenario de su independencia, motivo por el cual el estado peruano emitió normatividad específica, con la finalidad de lograr la excelencia educativa en todos los niveles de educación básica regular, técnico, universitario y post grado, que orientan al estado peruano en las pautas que deben seguir las instituciones educativas para lograr la calidad en los servicios que prestan, tanto académica como administrativamente; poniendo énfasis en el desarrollo de la educación superior universitaria publica de provincia; específicamente en este nuevo milenio, acciones que tienen como firme intento la mejora de la calidad de los procesos que involucran el desempeño docente de la docencia universitaria de provincia.

Cárdenas (2015), refiere que los estudios que, tiene por finalidad evaluar si se ha logrado implementar y mejorar la calidad de los procesos tanto académicos como administrativos, que se desarrollan en las escuelas profesionales y el desempeño docente en las universidades públicas de provincia.

Organización de las Naciones Unidad para la educación ciencia y cultura (2019), organismo internacional en coordinación con el ministerio de educación, como entidad representante del estado peruano y líder en el tema educativo a nivel nacional, ha venido desplegando acciones de fortalecimiento en el tema de calidad, acciones en las cuales viene participando la docencia universitaria, personal administrativo y estudiantes; a quienes se les viene inculcando el conocimiento de los componentes para valorar la calidad de modo exhaustivo, así como también en el tema del desempeño docente en todos sus aspectos, desde la elaboración del silabus hasta el desempeño en aula.

landing.edmachina (2019), publicación que explica detalladamente, el significado de la implementación del sistema de gestión de calidad, en las universidades públicas; de provincia; inicialmente motivado por que la razón de ser de las universidades es la académica; dentro de ello podríamos mencionar que los procesos académicos y administrativos que se llevan a cabo dentro de la institución, sugieren ingresar a un proceso de estudio para determinar cuáles son los cuellos de botella que se presentan y empezar a mejorar paulatinamente los mismos, todo ello con la finalidad que, con el transcurso del tiempo se constituyan en procesos más agiles y con ello satisfacer a 
los estudiantes; igualmente, se tiene como finalidad también verificar la situación del desempeño docente en la educación superior universitaria publica de provincia, ambas situaciones han sido objeto de muchas controversias en todos los ámbitos todo ello debido principalmente a las dificultades que presentan los egresados en lograr un puesto de trabajo; todo lo cual lleva a confusión en vista de la variedad de las actividades en el ámbito académico como administrativo y el conflicto de obtener trabajo rápido por parte de los egresados de aquéllas instituciones que se consideran con prestigio, como certeza de la calidad de una institución educativa superior.

Superintendencia Nacional de Educación Superior Universitaria (2016), institución encargada de la supervision de la educacion superior universitaria, quien nos manifiesta que la formación superior universitaria de provincia está delimitada a los procesos que el estado peruano viene implementado con motivo del licenciamiento y acreditación institucional, con la finalidad de unificar criterios y mejorar la calidad educativa en la educación superior en nuestro país, con incidencia en el tema de la educación superior universitaria, cambio social, cambio tecnológico y cultural, desde el tema de la calidad, con el objetivo de lograr alcanzar niveles de calidad en la educación a nivel de latino américa.

MONTENEGRO ORDONEZ (2020), quien manifiesta en su publicación que, la formación profesional de los estudiantes pende de la columna de la docencia en la educación superior pública de provincia, esperando un excelente trabajo por parte de la docencia universitaria; y consiguientemente el aumento de la calidad de los egresados de las universidades públicas de provincia.

Gaceta (2018), informe periodístico que nos precisa que la mejora de la gestión de la calidad, está ligada con el incesante cambio en los temas académicos, así como también con las técnicas de gestión. Siendo los criterios de la calidad en este estudio, tres directrices, las cuales son:

- Gaceta (2018), precisa que el docente universitario es un individuo, que nos permite valorar lo que se va a encontrar de las personas, tenemos que conocer al docente no sólo académicamente, sino en su desempeño en el aula y a la potestad de los saberes previos en clase sino también buscar muchos aspectos afables y de temperamento.

- Gaceta (2018), refiere que la valoración de la docencia universitaria de universidades públicas en provincia, como parte integral del currículo de la misma, refiere que el 
silabo es una experiencia desarrollada, a través de muchos métodos, configura la agilidad pedagógica del docente universitario, quien es el dispositivo activo que se traduce en la práctica.

- Gaceta (2018), manifiesta que la docencia universitaria publica de provincia, a manera de experiencia social es un combinado, que se despliega en la clase por un docente con un conjunto de alumnos que repercute los límites de lo individual. Es así que a los docentes corresponderán ser facilitadores de la enseñanza y tutores; los estudiantes, deberán ser más diligentes comprometidos en su proceso enseñanza aprendizaje curricular evaluando en el estudiante el aprendizaje demostrativo; debemos formar estudiantes con una visión completa de las ciencias, responsabilidad interdisciplinaria, actualización permanente en los esquemas educativos; así como también la formación permanente de la docencia, desarrollo completo de contenidos cognoscitivos, sinapismo del pensamiento crítico y sentido de responsabilidad social.

Escribano (2018), indica que el motivo por el cual se requiere instituir la calidad del desempeño docente, contrapuesto con la gestión de la calidad en las universidades públicas de provincia; conocer y entender las discrepancias en la aptitud de acuerdo con la experiencia de los catedráticos, así como, a las marcadas diferencias al tipo de contrata que tienen y régimen de dedicación de la docencia nombrada. La presente investigación se desarrolla en la universidad nacional de san Antonio abad del Cusco, específicamente en la escuela profesional de economía.

\section{Objetivos del estudio}

- Determinar en qué manera influye la gestión de la calidad sobre el desempeño docente de la escuela profesional de economía de la Universidad Nacional de San Antonio Abad del Cusco

\section{Objetivos específicos}

- OE1: Determinar cómo la gestión de la calidad influye en las capacidades pedagógicas didácticas en la escuela profesional de Economía de la Universidad Nacional de San Antonio Abad del Cusco.

- OE2: Determinar cómo la gestión de la calidad influye en la responsabilidad en el desempeño de sus funciones laborales de la escuela profesional de Economía de la Universidad Nacional de San Antonio Abad del Cusco. 
- OE 3 Determinar cómo la gestión de la calidad influye en los resultados de su labor educativa de la escuela profesional de Economía de la Universidad Nacional de San Antonio Abad del Cusco.

\section{MATERIALES Y METODO}

\section{Materiales}

Ministerio de Educación (2012), precisa los instrumentos que se utilizaron en el presente trabajo de investigación son: encuestas y entrevistas, enmarcadas en el tema de desempeño docente, la misma que incluye horarios de acuerdo a las funciones que realizan los docentes de la institución: Docencia-Enseñanza, Docencia-Aprendizaje, Tutorías, Investigación, Gestión Académica y Formación Académica. Con el soporte de los mismos se formularon las preguntas que están clasificadas en directas y cerradas, y de opción múltiple, también se utilizan entrevistas paralelas divididas en dos grupos para docentes y estudiantes. Prosiguiendo con la investigación, se muestra la definición de cada indicador:

- Ministerio de Educación (2012), indicador Docencia-Enseñanza, en la publicación referida al marco del buen desempeño docente, describe cuales son las habilidades y recursos pedagógicos que utiliza el docente para compartir sus cursos.

- Ministerio de Educación (2012), indicador Docencia-Aprendizaje. en el marco del buen desempeño docente, describe cuales deben ser las habilidades de investigación y supervisión de compromisos, apreciación, retroalimentación, colaboración, conducta y estimulación a los alumnos.

- Ministerio de Educación (2012), indicador Tutorías. el marco del buen desempeño docente, refiere las habilidades que maneja el docente para trabajar con estudiantes y la labor de tutoría que se realiza con ellos, asesoría en tesis, propuestas particulares de estudiantes, comprometido con el servicio social, experiencia pre profesional, consejero de carga académica, de problemas en su desempeño correcto y en las iniciativas de titulación.

- Ministerio de Educación (2012), indicador Gestión Académica, precisa que podríamos clasificar la gestión académica en los siguientes grupos: comisiones de revisión de documentos para grados y títulos, de revisión de documentos para convalidaciones y homologaciones, labor no lectiva de la docencia universitaria es su participación en comisiones académicas y otras comisiones específicamente 
conformadas para el proceso de acreditación entre las que tenemos: comisión para el examen, diseño y valoración de los nuevos planes de estudio, activa participación en proyectos de investigación fondo especial de desarrollo universitario, que permiten la vinculación entre el docente con los estudiantes; además tenemos la combinación y supervisión de transmisiones educativas de investigación.

- Ministerio de Educación, (2012) indicador Formación académica, en el marco del buen desempeño docente refiere la formación docente en eventos de posgrado, capacidades o cursos para renovar la materia que se imparte.

\section{Siendo la idea principal fortalecer los índices de aptitud para el ejercicio de los docentes en las materias que regenta.}

GOMEZ (2019), precisa en su publicacion que con el afán de instaurar los índices de aptitud del desempeño docente de los profesores de universidades públicas de provincia que se encuentran licenciadas, se transformaron en réplicas, apreciaciones, lo cual se logró consentir en un valor a cada consulta a los involucrados en la investigación:

\section{Participantes}

Salas (2020) manifiesta que para determinar la población considerada para el siguiente trabajo de investigación, estuvo conformada por 25 catedráticos nombrados y contratados, 720 estudiantes, de una escuela profesional de una universidad pública de provincia San Antonio Abad del Cusco; para el proceso de selección de la población se efectuó de la siguiente manera: docentes nombrados y docentes contratados, personal administrativo, población estudiantil la misma que está conformada por 720 estudiantes elegidos de modo no probabilístico, tanto la docencia como los estudiantes deben estar cursando asignaturas con los docentes participantes, con la finalidad de lograr un estudio uniforme de las variables, para con ello lograr los resultados esperados. Es necesario precisar además que las encuestas se aplicaron utilizando el google meet.

\section{Procedimiento}

- Instituto Nacional para la evaluación de la educación - México (2017), precisa el diseñó y la forma de la encuesta encerrando los indicadores mencionados.

- Instituto Nacional para la evaluación de la educación - México (2017), precisa el diseñó de las entrevistas, tomando como referencia los indicadores del desempeño docente y conocer cuál es la opinión acerca de los mismos.

- Instituto Nacional para la evaluación de la educación - México (2017), precisa el 
diseño del entrenamiento a estudiantes del centro federado en la aplicación de encuestas online.

Se realizó posteriormente la clasificación y análisis estadístico de los datos.

\section{METODOLOGIA}

La metodología propuesta para el presente trabajo de investigación es el siguiente:

Monge (2011), manifiesta que el proceso de la investigación tiene carácter ex post facto, lo cual significa que la investigación se realizara después del hecho, en este tipo de diseño la investigación plantea que la validación de la hipótesis se realiza cuando ya ha sucedido; se trata en realidad de una búsqueda de las causas que lo han ocasionado, en un pasado remoto, podríamos indicar además que hipotéticamente si el suceso no se ha podido producir en realidad no conviene hacerlo.

Cancela, Cea, Galindo, \& Valilla (2010), manifiesta que los estudios post facto engloban la mayoría de los métodos de investigación descriptiva, equivale a decir, que casi todos los estudios no son de tipo experimental, puesto que sus manifestaciones ya han ocurrido, equivale a decir que el hecho ya ocurrió (variable independiente), y se observan luego los efectos de las variables dependientes.

Cancela, Cea, Galindo, \& Valilla (2010), ademas indica que las investigaciones ex post facto tienen limitaciones entre las cuales podemos mencionar:

- Cancela, Cea, Galindo, \& Valilla (2010), manifiestas que la incapacidad de controlar directamente a las variables independientes, lo cual genera desconfianza.

- Cancela, Cea, Galindo, \& Valilla (2010), indica que no se puede realizar asignaciones aleatorias.

- Cancela, Cea, Galindo, \& Valilla (2010), precisa que el riesgo con las interpretaciones erróneas por la falta de control Bisquerra.

Monge (2011), quien precisa que las investigaciones causales tienen como función descubrir y recabar las bases de la información, lo cual va a permitir obtener el resultado del estudio, la formulación de la hipótesis teniendo como corolario familiarizar al investigador con un objeto que le resultaba extraño.

Monge (2011), precisa que las investigaciones exploratorias son las que tienen el primer acercamiento al problema de la investigación la cual se tiene que estudiar y conocer el tema y familiarizarnos.

Miralles, Alfagene, \& Rodriguez (2014), precisan que, para luego proceder con la 
investigación, se trabaje la muestra aleatoria de personal docente, administrativo y estudiantes de la escuela profesional de economía, habiendo entonces concertado la cita con cada uno de los docentes seleccionados se procedió a realizar la entrevista, con el siguiente orden:

\section{Docentes}

El entrevistado manifestó que es docente universitario hace 32 años y tiene la condición de docente nombrado a dedicación exclusiva en la escuela profesional de economía, regentando varias materias en la escuela, por ejemplo economía agraria, costos y presupuestos entre otros, sin embargo manifiesta que, con la promulgación de la nueva ley universitaria, y el ingreso de las universidades públicas y privadas a este nuevo enfoque, la metodología de trabajo ha cambiado drásticamente en la escuela, el tránsito a la nueva normatividad impuesta por el estado peruano, ha implicado cambios en los currículos de estudio, cambios en los procedimientos para la obtención de grados y títulos, más aun con la dación de la nueva normatividad referida a los procesos de licenciamiento y acreditación de las universidades a nivel nacional, que significa mejorar procesos, al respecto manifiesta también que en la escuela profesional de economía se ha conformado comisiones para unificar criterios todo ello con la finalidad de lograr un avance sostenido de los procesos y contar con la participación de todos los actores del proceso, al respecto es necesario resaltar que para los docentes "es muy complicado y duro el tránsito de la educación convencional a la educación digital".

Es así que otro docente de la escuela profesional de economía, manifiesta que es docente de la escuela hace 35 años, siendo la especialidad de la misma la asignatura de microeconomía y macroeconomía; y ha conformado varias comisiones de trabajo relacionados al tema de implementación del sistema de gestión de la calidad y desempeño docente, manifestando explícitamente que con relación a la implementación del sistema de gestión de calidad poco o nada se ha implementado, situación que se ha agudizado con la pandemia de la COVID 19, sin embargo como aporte a la escuela profesional de economía se ha indagado con relación a los procesos que se llevan a cabo en la escuela profesional y si ellos pasaron por el proceso de normalización para su funcionamiento, sin embargo es necesario precisar que, estos documentos no forman parte del sistema de gestión de la calidad de la institución, con relación al desempeño docente refiere que se elaboraron cuestionarios dirigidos a los estudiantes para evaluar el desempeño docente de 
la docencia de la escuela de economía, sin embargo los mismos no fueron validados aun así se llevó adelante la encuesta a los estudiantes. Precisa además que, desde la dación de la nueva ley universitaria el proceso de tránsito a la misma ha reflejado para la docencia que tiene muchos años trabajando en la docencia a la era digital ha significado mucha dificultad y proceso de acondicionamiento a la realidad virtual.

Es así que la entrevista más interesante del trabajo de campo se realizó a un docente que viene laborando 10 años en la escuela profesional de economía, quien manifiesta que ha desempeñado varios cargos tanto académicos como administrativos en la universidad nacional de san Antonio abad del cusco, entre los cuales se puede mencionar que se desempeñó como director de la dirección de acreditación de la escuela profesional de economía a la fecha, y se estuvo elaborando documentación que servirá de evidencia para el proceso de acreditación de la escuela profesional de economía, sin embargo es necesario indicar que la documentación elaborada no ha sido hecha tomando como referencia establecido en los sistemas y programas del sistema de gestión de la calidad, refiere además que se ha estado laborando conjuntamente que los grupos de interés, que se encuentran en la escuela profesional como son los estudiantes representados por el centro federado y los estudiantes que pertenecen al tercio estudiantil; todo este trabajo se quedó en stand by por la pandemia de la COVID 19, motivo por el cual no se ha proseguido con el trabajo indicado. Precisa además que a pesar de estar laborando ya hace algún tiempo en la escuela antes indicada el proceso de transición a la nueva normatividad impuesta por el estado la ha encontrado interesante y adecuada al nuevo milenio, indicando además que aún no se ha establecido los indicadores para evaluar el desempeño docente, sin embargo, se viene evaluando el desempeño docente con los indicadores referidos por la autoridad universitaria.

Al ser entrevistado un docente que a pesar de contar con más años en la escuela profesional nos indica que ingreso a laborar a la institución desde la condición de la ayudantía de catedra motivo por el cual tiene más años laborando en la institución, indica además que ha desempeñado cargos en el tema docente como administrativo hasta hace algunos años atrás, manifiesta igualmente que el proceso de transición a la nueva normatividad impuesta por el estado peruano ha significado para los docentes jóvenes no mucho problema, sin embargo ha representado un cambio notable para los docentes que ya tienen laborando más de 30 años en la institución, la imposición de un nuevo currículo 
la misma que no necesariamente contiene los cursos que se venían dictando antes de la nueva ley universitaria, agregando además a lo citado el tema del cambio de los procedimientos para los trámites administrativos, y además con motivo de la pandemia de la COVID 19, el proceso de transición del sistema presencial convencional a la virtualidad ha significado para muchos docentes un cambio drástico innovador pero de difícil adaptación.

Es importante señalar que, con el tema de la pandemia de la COVID 19, los servidores administrativos de la escuela profesional han disminuido notablemente, antes del inicio de la pandemia se tenía 3 servidores administrativos que laboraban en la escuela profesional, a la fecha se cuenta solo con una persona que atiende la dirección de la escuela profesional, quien manifiesta que fue asignada a laborar en la escuela profesional un mes antes del inicio de la pandemia, y a la fecha viene realizando trabajo mixto sin embargo a la fecha no ha participado de proceso alguno de acreditación en la escuela profesional.

Universidad de Valencia (2007), en su estudio de investigacion precisa que, uno de los grupos de interés a los cuales se les debe asignar un lugar preponderante en la investigación son los estudiantes que evalúan los procesos que se llevan a cabo en la escuela profesional como clientes y son ellos los que determinan en muchos casos con sus apreciaciones el verdadero sentido de la investigación.

Se entrevisto a un estudiante egresado hace un semestre de la escuela profesional de economía, quien manifestó que en su promoción no se ha sentido mayormente el proceso de acreditación en la escuela, porque muchas de las reuniones de trabajo convocadas para tal fin fueron limitadas en su participación a los estudiantes que conformaban el centro federado o el tercio estudiantil, se esperaba convocatorias más abiertas para tal fin. Con relación al desempeño docente precisa que los docentes que vienen laborando más años en la escuela, no modifican significativamente el contenido de sus sílabos para los cursos sobre todo de especialidad, situación que no es similar con la docencia contratada sílabos ajustados a la realidad económica del país, precisa además que no se les proporciono cuestionarios para evaluar el desempeño docente. Añade además que en los procesos administrativos se tiene mucha demora especialmente en las comisiones de calificación de documentos, lo cual conlleva en muchos casos a la perdida de oportunidades laborales por la falta de los bachilleratos y títulos en menor tiempo, indica además que el trámite 
de bachillerato que realizo a pesar de haberlo realizado de manera virtual apenas había egresado de la escuela profesional demoro 6 meses.

Es así que otro estudiante que tiene la condición de recién egresado manifiesta que la falta de información por parte de la docencia y el desconocimiento de los documentos de gestión ocasiono que surjan inconvenientes al momento de egresar de la escuela profesional, en sus trámites referidos a la homologación de asignaturas, para poder acceder a las practicas pre profesionales, así como también en la demora de la obtención del bachillerato por inconvenientes en los tramites especialmente en las comisiones de revisión de documentos, refiere además que a la fecha de egreso no se les circulo cuestionario alguno para la evaluación del desempeño docente, precisando además que la docencia que tiene con muchos años de servicio no utiliza en el desarrollo de sus clases la tecnología como por ejemplo las pizarras virtuales, ecran, retroproyectores, mucho menos conocían el funcionamiento de las plataformas virtuales para el desarrollo de asignaturas, situación diferente que observo con docentes de muy buena calidad y docentes que no cuentan con capacitación y especialmente en el tema de metodología de enseñanza, que aun siendo muy buenos profesionales, no tienen metodología para llegar al estudiante.

En la entrevista realizada a los estudiantes que aún se encuentran cursando la escuela profesional, la opinión versada es la siguiente:

Estudiante de los últimos semestres de la escuela profesional manifiesta que en lo referido al desempeño docente opina que la docencia de la escuela son muy buenos profesionales en las especializaciones que eligieron, sin embargo en la metodología de la enseñanza no tienen muchas aptitudes para realizar el trabajo docente, el desempeño docente no es muy aceptable para el momento que el estudiante egresa de la escuela profesional, y tiene que competir con estudiantes egresados de otras universidades que tiene currículos actualizados, los mismos que se encuentran enmarcadas en procesos de acreditación de facultades, refiere además que los trámites administrativos aún no se encuentran enmarcados en lo estipulado en la nueva normatividad impuesta por el estado.

Refiere otro estudiante que se encuentra cursando el sexto semestre de la escuela profesional y que a la fecha desconoce cuáles son los procedimientos que debe realizar por ejemplo para poder acceder a las practicas pre profesionales, muchos menos de los tramites de homologación y demás que colaboran con el tema de la obtención del grado 
académico, y posterior título profesional. Con relación al desempeño docente refiere que los docentes son excelentes profesionales sin embargo muchos de ellos carecen de habilidades pedagógicas para llegar de manera propicia a los estudiantes y colaborar con la formación profesional, agrega además que muchos de los docentes en el proceso de virtualización de la enseñanza todo ello producido por la pandemia de la COVID 19 les ha resultado muy complicado el ingreso a la enseñanza virtual, muy difícilmente manejan las plataformas virtuales.

Sistema Nacional de Evaluación, Acreditación y Certificación de la Calidad Educativa (2018), explica pausadamente la implementación de los procesos para los sistemas y programas referidos en los estándares para lograr tanto el licenciamiento como la acreditación de universidades se encuentran aún en proceso de implementación, considerando además que dicho proceso se debe realizar a nivel institucional.

Jofré (2009), refiere que con relación al proceso del desempeño docente, podemos precisar que es opinión de casi todos los entrevistados que sus docentes son muy buenos profesionales, sin embargo carecen de habilidades referidas a la pedagogía para con ello contribuir con la mejor formación profesional; la docencia no maneja plataformas virtuales, lo cual no colabora con el aprendizaje virtual como se viene dando en la actualidad todo ello provocado por la pandemia de la COVID 19; e incluso en la presencialidad de las clases tampoco utilizan las herramientas tecnológicas que les proporciona la institución como son: pizarras electrónicas, ecran y demás; lo cual ha dificultado enormemente el proceso de ingreso a la virtualidad y al desenvolvimiento de las clases virtuales. Es entonces que el desempeño docente incluye en su quehacer los proyectos e iniciativas que involucran directamente el trabajo de la docencia universitaria en aula.

\section{RESULTADOS}

Congreso de la República, (2014) determino los estándares para el desempeño docente en las universidades públicas en nuestro país está delimitado por la promulgación de la ley universitaria $\mathrm{N}^{\mathbf{0}}$ 30220, y a su vez la exigencia del estado peruano referida a los métodos de licenciamiento y acreditación de los establecimientos educativos universitarios, cuyo significado aparentemente compartido es motivo de mucha controversia.

La población de este estudio se conoció que el $25 \%$ son docentes nombrados con una 
antigüedad mayor a 30 años. En todo lo que se refiere al tema de formación académica, se halló que el grado académico de maestría fue el más elevado con un $41 \%$, el título académico un $12 \%$, la particularidad el $22 \%$ y el más bajo el de doctorado con un $11 \%$. Universidad Autónoma Indigena de México (2016), precisa que desde la percepción de los estudiantes de las universidades públicas de provincia el desempeño docente, se ve reflejada en el escaso conocimiento que los estudiantes de las funciones que deben ejecutar los docentes de las universidades públicas de provincia, lo cual podría deberse a que la población estudiantil ha sobrepasado el límite de capacidad establecido dentro de los estándares establecidos por el estado peruano para el licenciamiento institucional; lo cual limita al docente para poder establecer contactos personales con los estudiantes y desempeñar sus funciones como la institución lo solicita, igualmente a los estudiantes se les invita a participar en diversas actividades no curriculares, programas deportivos, e invitan a ser parte de los grupos de investigación que se fomentan en la Universidad; actividades con las que se percibe que las jefaturas de departamento, los docentes y estudiantes conocen muy poco del proceso de autoevaluación y los procesos que conlleva la misma.

No se mostraron discrepancias de disposición por rango de antigüedad, esto tal vez simbolice, que, de acuerdo a las políticas institucionales actuales, se les exige a los catedráticos cumplir por un lado de mayor productividad, sin considerar al tipo de su contrata y de su experiencia, para amparar un patrón de producción y de calidad en la institución.

En conclusión, los niveles de calidad fueron significativamente pequeños a los horizontes teóricos determinados a través de clases remotas; se debe reflexionar en los factores de reforma para el cumplimiento de los nuevos catálogos de cursos de cada semestre, el involucramiento en la correcta utilización de los mismos obedeciendo su categoría laboral y la oportunidad de los elementos que constituyen el quehacer académico.

Los docentes, por el nuevo rol que les requiere en la universidad nacional de provincia, deben abocarse en la sistematización académica institucional, se deben nivelar las otras funciones que también son significativas para lograr la calidad oportuna.

Las nuevas capacidades para lograr el cambio rápido exigirán a los docentes a ser elásticos, acomodar y concebir en un ambiente cambiante; a aprender, desaprender y reaprender; a ser efectivos líderes, creativos y competentes de avanzar a la evolución 
de las efemérides

En el desempeño de estándares mínimos de calidad vinculados a los varios ejemplos de Instituciones de Educación Superior universitaria a manera una situación impostergable para el ejercicio de todas las funciones académicas, la universidad está buscando entre sus docentes es la formación doctoral.

Uno de los factores determinantes principales es el vínculo laboral bajo el cual laboran los docentes universitarios, que marca diferencia en el monto de las remuneraciones que perciben, los docentes universitarios de provincia nombrados perciben remuneraciones determinadas por la normativa de la ley universitaria 30220, sin embargo los docentes universitarios de provincia contratados tienen dos alternativas de contrato: uno con la institución y el contrato más atractivo económicamente hablando es el que se realiza con el ministerio de educación, que otorga remuneraciones equivalentes a las otorgadas con la ley 30220 aun incluso con menor régimen de dedicación.

A lo cual se debe agregar que con la declaración de estado de emergencia sanitaria, por el ejecutivo peruano, debido a la pandemia de la COVID 19, el proceso de enseñanza aprendizaje ha pasado de la presencialidad a la virtualidad, la academia no contaba con plataformas virtuales necesarias para el desarrollo de clases virtuales, aulas virtuales, a la fecha aún en muchas universidades no se tiene plataformas virtuales propias para el desarrollo de las actividades académicas, lo cual disminuye notablemente la calidad educativa, por otro lado la manifestación de los estudiantes que los docentes son excelentes profesionales mas no tienen la preparación suficiente como para desempeñarse como docentes pues carecen de pedagogía, en muchos caso agregar además que no manejan tecnología informática para los procesos de enseñanza aprendizaje.

Con relación al proceso de acreditación de la escuela profesional se han venido desarrollando actividades tales como reuniones, que no están siendo enmarcadas dentro de la propuesta de implementación del sistema de gestión de la calidad, agregando a ello que desde la dación de la nueva ley universitaria muchos de los procesos que se venían desarrollando en la academia han debido ser acomodados al nuevo marco legal impuesto por el ejecutivo, sin embargo a la fecha no se ha podido ajustar estos procesos a lo establecido en la normatividad vigente, lo cual dificulta enormemente el tema de 
la fluidez de la documentación agregando a ello que las comisiones conformadas por la docencia universitaria, no cumplen con los plazos establecidos, contribuyendo con ello a la demora de la obtención de grados y títulos por parte de los egresados, así como también demora en los trámites administrativos de homologaciones, obtención de constancias y demás que se emiten en la escuela profesional.

En el tema administrativo los procesos aún no han sido establecidos mucho menos normalizados y haber establecido la ruta más óptima para la atención de los mismos.

Se pretende lograr que los rangos de calificación a obtener sean la respuesta a los docentes entrevistados para lograr la diferencia con el prorrateo teórico de las categorías de evaluación esperados.

\section{DISCUSION}

Son entonces los principios teóricos que se plantean debemos conocer al docente universitario como persona, el cual va a ser evaluado desde la implementación de las nuevas curriculas las cuales se encuentran inmersas en la práctica social desde la idea de la calidad que van de la mano con una serie de horarios que constituyen el ser, el hacer y él debe ser de las universidades públicas del interior de nuestro país, lo cual permitirá al docente reflexionar a través de una entrevista en lo importante que será su participación en la causa de autoevaluación para la refrendación. En este argumento los indicadores de calidad que se refieren se deben indicar que los docentes universitarios de universidades públicas de provincia se encentran con un nivel de calidad mucho mejor de lo esperado.

Debemos recalcar que el conocimiento de la calidad del docente universitario de provincia, observada desde el punto de vista de la Jefatura del Departamento Académico de cada escuela profesional, el tema de la investigación, gestión y la formación académica en contrapuesta con labores cotidianas que ejecuta el docente universitario en clase tales como docencia enseñanza, docencia aprendizaje y la labor de tutoría podemos indicar que se percibe que la docencia piensa que no existe cultura organizacional en los procesos de evaluación de funciones, motivo por el cual se tiene más comprensión de los conocimientos administrativos que de la práctica docente.

Las Jefaturas de Departamento de las Escuelas profesionales conocen más las funciones del docente universitario de provincia y de alguna manera controlan el proceso que se debe realizar para lograr la investigación, formación académica lo cual 
debe ir de la mano con las actividades que el docente universitario de provincia debe realizar en aula procesos como docencia enseñanza, docencia aprendizaje y tutorías, para el desarrollo de las mismas se debe implementar y hacer parte de la vida universitaria tanto del docente del estudiante y del personal administrativo; de la cultura de evaluación y supervisión en la institución, durante el proceso de investigación se ha percibido que los docentes tienen más comprensión de los procesos administrativos que de la misma función docente.

Desde la percepción de los estudiantes de las universidades públicas de provincia el desempeño docente, se ve reflejada en el escaso conocimiento que los estudiantes tienen de las funciones que deben ejecutar los docentes de las universidades públicas de provincia, lo cual podría deberse a que la población estudiantil ha sobrepasado el límite de capacidad establecido dentro de los estándares establecidos por el estado peruano para el licenciamiento institucional; lo cual limita al docente para poder establecer contactos personales con los estudiantes y desempeñar sus funciones como la institución lo solicita, igualmente se invita a los estudiantes a participar en diversas actividades no curriculares, programas deportivos, y ser parte de los grupos de investigación que se fomentan en la Universidad; actividades con las que se percibe que las jefaturas de departamento, los docentes y estudiantes conocen muy poco del proceso de autoevaluación y los procesos que conlleva la misma.

Es entonces que partiendo con los principios hipotéticos planteados en el presente estudio se evalúa al el catedrático como persona, la valoración a la enseñanza como parte del silabus universitario, la docencia como experiencia social agrupando la idea de la calidad así como la unión de varios itinerarios, con un régimen de vínculos entre diferentes factores que forman el ser, el hacer y el deber ser lo que permitiría al docente por medio de una entrevista recapacitar en su oportuna práctica y anunciar rápidamente en el transcurso de autoevaluación.

No se mostraron discrepancias de disposición por rango de antigüedad, esto tal vez simbolice, que, de arreglo a las políticas institucionales actuales, se les exige a los catedráticos cumplir con un lado de mayor productividad, libre del tipo de su contrata y de su experiencia, para amparar un patrón de producción y de calidad dentro de esta erudición institucional.

En conclusión, aun y que los niveles de calidad inmersos fueron elocuentemente 
pequeños a los horizontes teóricos determinados a través de los itinerarios a distancia, no precisamente envuelve a que los catedráticos participantes carguen a cabo una labor defectuosa. Se debe reflexionar los factores de reforma para el cumplimiento de sus nuevos catálogos de cursos de cada semestre, el involucramiento en la correcta utilización de los mismos obedeciendo de su categoría laboral y la oportunidad de los elementos que constituyen el quehacer académico.

Los catedráticos, por el nuevo rol que les solicita apadrinar en la universidad nacional de provincia, se han abocado en la clasificación académica corporativa, que a nivelar las otras funciones que también se cavilan significativos para conservar una calidad oportuna. Las nuevas capacidades impuestas para acomodar al cambio rápido e invariable exigirán a los expertos en instrucción a ser elásticos, acomodar y concebir un ambiente tornadizo; a aprender, desaprender y reaprender; a ser efectivos líderes, creativos y competentes de avanzar a la evolución de las efemérides

En el desempeño de estándares mínimos de calidad ligados a los varios ejemplos de Instituciones de Educación Superior universitaria a manera una situación impostergable, para el ejercicio de todas las presentaciones de las similares, la universidad está originando entre sus docentes el horizonte de formación deseable de doctorado

Una de las determinantes principales es el vínculo laboral bajo el cual laboran los docentes universitarios lo cu|al hace la diferencia en el monto de las remuneraciones que perciben, los docentes universitarios de provincia nombrados perciben remuneraciones determinadas por la normativa de la ley universitaria 30220, sin embargo los docentes universitarios de provincia contratados tienen dos alternativas de contrato: uno con la institución y el contrato más atractivo económicamente hablando es el contrato que se realiza con el Ministerio de Educación, que otorga remuneraciones equivalentes a las otorgadas con la ley 30220 aun incluso con menor régimen de dedicación.

\section{REFERENCIAS BIBLIOGRAFICAS}

Cancela, Cea, Galindo, \& Valilla. (2010). http://files.investigacion-educativa9. Obtenido de http://files.investigacion-educativa9.: http://files.investigacioneducativa9.webnode.es/20000002828c3a29c05/Estudios\%20comparativos\%20casuales\%20o\%20ex\%20post $\% 20 \mathrm{fa}$ 
cto.pdf

Cárdenas, N. (2015). https://www.tdx.cat/. Obtenido de https://www.tdx.cat/: https://www.tdx.cat/bitstream/handle/10803/384556/necs1de1.pdf?sequence=1

Congreso de la República. (2014). http://www.minedu.gob.pe. Obtenido de http://www.minedu.gob.pe:

http://www.minedu.gob.pe/reformauniversitaria/pdf/ley_universitaria.pdf

Escribano,

H. (2018).

https://www.redalyc.org/.

doi:https://doi.org/10.15517/revedu.v42i2.27033

Gaceta. (2018). https://www.inee.edu.mx/. Obtenido de https://www.inee.edu.mx/: https://www.inee.edu.mx/wp-content/uploads/2018/12/G10ES.pdf

GOMEZ, L. F. (2019). http://www.scielo.org.pe/. Obtenido de http://www.scielo.org.pe/: http://www.scielo.org.pe/scielo.php?script=sci_arttext\&pid=S2307-

79992019000200019\&lng=es\&nrm=iso>.

ISSN

2307-7999.

http://dx.doi.org/10.20511/pyr2019.v7n2.255

Instituto Nacional para la evaluación de la educación - México. (2017). https://www.inee.edu.mx/. Obtenido de https://www.inee.edu.mx/: https://www.inee.edu.mx/wp-content/uploads/2019/08/P2A355.pdf

Jofré, G. (2009). https://www.tdx.cat/. Obtenido de https://www.tdx.cat/: https://www.tdx.cat/bitstream/handle/10803/5064/gjja1de2.pdf

landing.edmachina. (2019). https://landing.edmachina.com/. Obtenido de https://landing.edmachina.com/: https://landing.edmachina.com/es/ventajas-deun-enfoque-data-driven-en-la-gestion-

educativa?utm_term=gestion\%20de\%20universidad\&utm_campaign=LeadsSearch-

$1 \& u t m \_s o u r c e=a d w o r d s \& u t m \_m e d i u m=p p c \& h s a \_a c c=5882609403 \& h s a \_c a m=$ $12983945909 \&$ hsa_grp=124788627267\&hsa_a

Ministerio de Educación. (2012). Marco de Buen Desempeño Docente. Obtenido de Marco de Buen Desempeño Docente: http://www.minedu.gob.pe/

Ministerio de Educación. (2020). Consejo Nacional de Educación. Obtenido de Consejo Nacional de Educación: https://www.cne.gob.pe/

Miralles, Alfagene, \& Rodriguez. (2014). Dialnet-Investigacion. Obtenido de DialnetInvestigacion:

Dialnet-InvestigacionEInnovacionEnEducacionInfantil- 
684052.pdf

Monge, C. (2011). https://www.uv. $m x /$. Obtenido de https://www.uv.mx/: https://www.uv.mx/rmipe/files/2017/02/Guia-didactica-metodologia-de-lainvestigacion.pdf

MONTENEGRO ORDONEZ, J. (2020). http://www.scielo.org.pe/. Obtenido de http://www.scielo.org.pe/:

http://www.scielo.org.pe/scielo.php?script=sci_arttext\&pid=S1019-

94032020000100116\&lng=es\&nrm=iso>. ISSN 1019-9403. http://dx.doi.org/10.18800/educacion.202001.006.

Organización de las Naciones Unidad para la educación ciencia y cultura. (2019). https://repositorio.minedu.gob.pe/. Obtenido de https://repositorio.minedu.gob.pe/: https://repositorio.minedu.gob.pe/bitstream/handle/20.500.12799/6808/La\%20fo rmaci\%C3\%B3n\%20docente\%20en\%20servicio\%20en\%20el\%20Per\%C3\%BA $\% 20$ proceso\%20de\%20dise \%C3\%B1o\%20de\%20pol\%C3\%ADticas\%20y\%20g eneraci\%C3\%B3n\%20de\%20evidencias.pdf?sequence=1\&isAllowed=y

Salas, D. (11 de febrero de 2020). https://investigaliacr.com/. Obtenido de https://investigaliacr.com/: https://investigaliacr.com/investigacion/seleccion-dela-muestra-en-la-investigacion-cuantitativa/

Sistema Nacional de Evaluación, Acreditación y Certificación de la Calidad Educativa. (2018). https://occaa.unmsm.edu.pe/. Obtenido de https://occaa.unmsm.edu.pe/: https://occaa.unmsm.edu.pe/occaa/storage/uploads/files/2019\%20Explicaci\%C3 $\%$ B3n\%20de\%20est\%C3\%A1ndares\%20del\%20modelo\%20de\%20acreditaci\% C3\%B3n.pdf

Superintendencia Nacional de Educación Superior Universitaria. (2016). https://www.gob.pe/4504. Obtenido de https://www.gob.pe/4504: https://www.gob.pe/4504-superintendencia-nacional-de-educacion-superioruniversitaria-que-hacemos

UNESCO - CEPAL. (2021). Desafios para la educación que ha traído la pandemia en américa latina y el caribe. Comisión Económica para América Latina y el Carine. Obtenido de https://www.cepal.org/

Universidad Autónoma Indigena de México. (2016). https://www.redalyc.org/. Obtenido 
de https://www.redalyc.org/: https://www.redalyc.org/pdf/461/46148194007.pdf

Universidad de Valencia. (2007). https://www3.uji.es/. Obtenido de https://www3.uji.es/: https://www3.uji.es/ betoret/Formacion/Evaluacion/Documentacion/La\%20eval uacion\%20estudiantes\%20en\%201a\%20ESuperior\%20UV.pdf 